\title{
Reversing the Fields: Do Group-Delivered Services Belong Closer to the Center of a Transformed Health Care System?
}

\author{
John Weeks
}

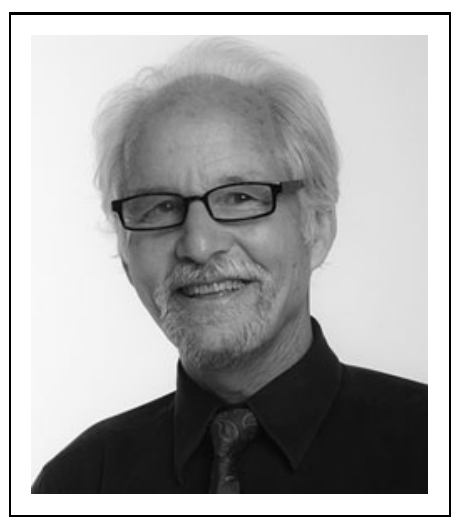

ture the necessary drama. A cochair of the Global Forum on Innovation in Health Professional Education at the U.S. National Academy of Sciences urged "blowing up the boxes" of the curriculum shaping present medical education. ${ }^{1}$ These comments followed a reflection of the other cochair that medicine "needs a transformation, and transformational change requires death ... and rebirth.",

Realistic assessment of the challenges from a paradigm shift toward a health approach are echoed at the top of health care policy and delivery in similarly disarming, or welcome, language-depending on one's perspective. A former administrator of the U.S. Center for Medicare and Medicaid Services who cofounded the value-based medicine driving Institute for Healthcare Improvement urged a global gathering of hospital leaders toward a "salutogenic" focus. This, he argued, would require taking steps "more radical than we have yet imagined."3 A chair of the American Hospital Association who was similarly reflecting on redirecting the mission of his member organizations from disease management to "creating health" admitted being stumped: "I don't think anyone yet knows the recipe."

This context frames the theme of this JACM Special Focus Issue on Innovation in Group-Delivered Services. A move to use of group medical visits from one-on-one care can be seen simply as an added seasoning to adorn the regular practice of medicine. Yet even here paradigm issues press in on all sides. Commitment to quality use of group medical visits breaks large and small boxes. Clinical educators need to teach new skills that include collaborating closely with other disciplines and working with teams. Practitioners must educate patients to the value of group, apply new skills while leaving the comfort and control of a one-on-one treatment room for a group environment in which their choices are observed and may be challenged by increasingly Internetempowered audiences. Challenges to administrators posed by groups range from space, check in, staffing, and phone protocols to scheduling and the barriers to appropriate payment.

Each of these becomes a broader systemic concern if one imagines that group-delivered services merit consideration not as seasoning but as staples in a transformed system where the focus shifts from masking or suppressing symptoms and managing disease to creating health or salutogenesis. Might fundamentally shifting substantial treatment-particularly of those with chronic conditionstoward group-delivered methods be essential ingredients of the recipe to reach what the radical reformers at the U.S. Veteran's Administration call the future state of "whole health"?

In medicine's “C-suites" (CEO, CMO, CFO), evidence available today for group-delivered integrative services would embroil them immediately in the most pivotal and revolutionary question: Is their business-nominally not-for-profit although it may be-fundamentally about the production and volume focus of a medical industry or the value and health focus of an organization that merits the title of health care system? Cost savings from three of the best researched of integrative health practices-each delivered through group visits - show remarkable diminution of the need for the services that are the medical industry's current staples:

- Multiweek group-delivered programs created by Dean Ornish and others to reverse heart disease found per capita savings - through foregone cardiovascular services-at $\$ 17,687$ per participant over a 3-year period. ${ }^{5}$

Editor-in-Chief, The Journal of Alternative and Complementary Medicine. 
- Multiweek group-delivered multimodal mindfulnessbased stress reduction programs developed by John Kabat-Zinn produced cost savings of $\$ 724$ per participant relative to usual care. ${ }^{6}$

- Multiweek group-delivered multimodal relaxationfocused programs developed by Herbert Benson and others were associated, in a retrospective examination of 4452 participants, with $43 \%$ lower use on average of pharmaceuticals, emergency room visits, hospital visits, and physician visits than in controls. ${ }^{7}$

If one questions whether the use of group-delivered medical services meets the threshold of box-breaking and radical, picture how unsettling the $43 \%$ reduction may be in the hospital C-suite where success is measured in revenues created from services that the group services are associated with radically reducing. One might wonder too at the response in the C-suite to a "Policy Recommendation" at the end of the study. The authors suggested that for the entire population such well-researched mind-body group-delivered behavior change programs "should perhaps be instituted as a form of preventative care similar to vaccinations or driver education." What might the impact be of such a public health innovation delivered through groups?

If one allows common sense and such present evidence to cut through the bondage of the normal cultural economics of one-on-one care, a case statement for pushing research, education, and practice toward expansive use of groups ripples down before one like a red carpet.

- Most of our challenges-in health and medical economics-are with chronic conditions.

- Creating health in people with virtually all such conditions requires central engagement with lifestyle changesthe behavioral determinants of health.

- Face-to-face teaching and even video or web-based exchange of information about such conditions and how to make changes is most efficiently delivered in a group environment.

- Adults learn better in interactive group environments than one on one.

- Engaging active practices to positively influence one's behavioral determinants - such as meditation, mindfulness, cooking kitchens, yoga, t'ai chi, and Zumbais fostered with and through groups.

- Group environments limit disabling influences of transference in one-on-one expert-to-patient environments. As group acupuncture proponent Michael Smith, MD, once opined: "If you want to empower patients, put more patients than providers in the room.",

- Evidence that social isolation is a significant contributor to chronic pain conditions suggests that the therapeutic medium of groups be part of the message of change. ${ }^{9}$

- To the extent that community centers can be the locus for change on social determinant that impact health, the use of these for group activities fosters connections to support such campaigns.

The appeal to the evidence-informed essential common sense in most of this list is acquiring a growing base of research support. Leaders of a functional medicine-focused integrative group program at the Cleveland Clinic report that their model is more rapidly shifting patient-centered outcomes scores than a team-based functional medicine model that is itself outperforming regular primary care. ${ }^{10}$ Some of the evidence is being offered up in this journal. A remarkable experience of a cancer center with acupuncture reported by Gentile and Yaguda found that switching from one-on-one treatment to group both increased income and access. The systematic review by Pariky et al. provides a look at key components.

A mentor in my personal journey toward considering the potential of groups was Eileen Stuart-Shor, $\mathrm{PhD}, \mathrm{RN}$ who worked in the 1980s and 1990s with Herbert Benson and others in a pioneering multiweek group-based programs that were shown to reverse the course of heart disease..$^{11}$ She described what became a familiar experience as she increasingly created opportunities to present their positive outcomes in conventional cardiology forums. In the summative tale, a fellow panelist would share outcomes from a new pharmaceutical agent that, while positive, showed less change than the group-delivered program she was reporting. Stuart-Shor observed repeatedly that regardless of the positive differential in favor of her groupdelivered medical intervention, attendees flocked to the other presenter asking for more information. Optimal use of groupdelivered medical services is box-breaking and radical (personal communication, 1999).

The transformative change toward group medical visits from one-to-one services is an equal opportunity challenge to conventional, complementary, and integrative practitioners. Despite the alignment of integrative, naturopathic, and functional medicine mission and values with this delivery method, professionals across all fields are products of health care educational systems that value and even glorify the sanctity of the one-to-one practitioner-patient relationship. Is it time to elevate the sanctity of the special value of the group as Geller explores in this issue-in medical treatment?

In recent years, promotion of group-delivered services at the Institute for Functional Medicine ${ }^{12}$ and by Integrative Medicine for the Underserved ${ }^{13}$ - a partner organization for this issue-appear to be leveraging change. A recent interview series on group-delivered services from James Mas$\mathrm{kell}^{14}$ that highlight the work at the Cleveland Clinic and of multiple authors in this issue includes evidence of changeif not yet of a tide fully turning to affirmatively explore all the potential to human health from innovation in, and implementation of, group-delivered services.

The good news for such an awakening is that subsets of health care leaders across multiple domains are increasingly aware that we need to be looking beyond present horizons for solutions that are more radical than are typically considered. The goal of this special focus issue was to help locate some contributions. JACM will continue to be interested in research on innovations in group-delivered services. The truism in paradigm shifts is that one cannot get out of a mess by reapplying the same tools that got one into it. Group-delivered medical services can be potent agents of change that hit a lot of the right notes.

\section{References}

1. Scrimshaw S. Global forum on innovation in health professional education. Meeting welcome. Online document at: www.iom.edu/Activities/Global/InnovationHealthProf Education/2015-APR-23/Day\%201/Welcome\%20and\%20 
Session\%20I/1-Welcome-Video.aspx, accessed May 13, 2015.

2. Cox M. Opening Comments [Closed Planning Session]. Global Forum on Innovation in Health Professional Education. Washington, DC, April 22, 2015. [Google Scholar].

3. Berwick D. "H4: The Nature of Transformation." Plenary presentation, Institute for Healthcare Improvement. December 13, 2013. Online document at: www.ihi.org/ resources/Pages/AudioandVideo/Don-Berwick-Forum-Key notes.aspx, accessed June 20, 2019.

4. Gaudet T, Kligler B. Whole health in the whole system of the veterans administration: How will we know we have reached this future state? J Altern Complement Med 2019; 25(S1):S7-S11.

5. Highmark Blue Cross Blue Shield. Dean Ornish program for reversing heart disease cost effectiveness summary. Online document at: https://www.ornish.com/wp-content/ uploads/Highmark-cost-analysis-2.pdf, accessed June 20, 2019.

6. Herman PM, Anderson ML, Sherman KJ, et al. Costeffectiveness of mindfulness-based stress reduction versus cognitive behavioral therapy or usual care among adults with chronic low back pain. Spine 2017;42:1511-1520.

7. Stahl JE, Dossett ML, LaJoie AS, et al. Relaxation response and resiliency training and its effect on healthcare resource utilization. PLoS One 2015;10:e0140212.

8. Smith M. Presentation on National Acupuncture Detoxification Association, Healthier Communities Best Practices Forum: East Meets West. Healthcare Forum, American Hospital Association, Honolulu, HI, November, 1999.

9. Mackey S. Learning health systems for optimized care and real-world research discovery. University of Washington School of Medicine, Department of Anesthesiology and Pain Medicine. June 5, 2019. Online document at: https://depts .washington.edu/anesth/administration/calendar/?trumba Embed=view\%3Devent\%26eventid\%3D129698621, accessed June 20, 2019.

10. Hyman M. Real world: integrative, functional and naturopathic clinical pilots in the era of value-based care. Panel presentation, Integrative Health Symposium. New York City, NY, February 22, 2019. Online document at: https:// www.ihsymposium.com/session/real-world-integrative-func tional-and-naturopathic-clinical-pilots-in-the-era-of-valuebased-care, accessed June 20, 2019.

11. Leserman J, Stuart EM, Mamish ME et al. Nonpharmacologic intervention for hypertension: Long-term follow-up. J Cardiopulm Rehabil Prev 1989;9:8

12. Institute for Functional Medicine. Ins and outs of the group visit model. Online document at: https://functionalmedicine coaching.org/ins-and-outs-group-visit-model, accessed June 20, 2019.

13. Lastad AT. Integrative Medical Group Visits at Community Health Centers. Hosted in the Toolkit webpage of Integrative Medicine for the Underserved. Online document at: https://im4us.org/toolkit/integrative-group-medicalvisits-at-community-health-centers, accessed June 20, 2019.

14. Group Visits Series, James Maskell host. Evolution of medicine-functional forum podcast. Online document at: https://functionalforum.com/category/podcast, accessed June 20, 2019.

Address correspondence to: John Weeks

johnweeks-integrator.com Seattle, WA 98116

E-mail: jweeks.jacm@gmail.com 